\title{
DISENTANGLING X-RAY REPROCESSING SITES IN EXO 0748-676
}

\author{
R. I. Hynes, ${ }^{1,2}$ C. A. Haswell, ${ }^{3}$ E. L. Robinson, ${ }^{1}$ K. Horne,${ }^{4}$ K. O'Brien, ${ }^{5}$ P. A. Charles, ${ }^{6}$ A. R. King, ${ }^{7}$ and \\ D. J. Rolfe ${ }^{7}$
}

\section{RESUMEN}

Presentamos los primeros resultados de un estudio coordinado en múltiples longitudes de onda de la estrella de neutrones de la binaria de baja masa de rayos X, EXO 0748-676 (UY Vol). Se obtuvieron datos UV rápidos, de rayos $\mathrm{X}$ y ópticos, incluyondo tanto información espectral como temporal. Discutimos cómo este estudio nos permite indagar sobre cuál es la distribución de temperatura dentro de la binaria y por lo tanto, sobre la geometría y la eficiencia de la irradiación de rayos $\mathrm{X}$.

\section{ABSTRACT}

We present first results from a coordinated multiwavelength study of the neutron star low-mass X-ray binary FXO 0748 676. Fast, UV, X-ray, and optical data were obtained including both spectral and timing information. We discuss how this study allows us to probe the temperature distribution within the binary and hence the geometry and efficiency of $\mathrm{X}$-ray irradiation.

\section{Key Words: BINARIES: CLOSE - STARS: INDIVIDUAL (EXO 0748-676)}

The importance of X-ray irradiation in low-mass $\mathrm{X}$-ray binaries is widely accepted; it may be responsible for much of the optical and UV flux, for stabilizing disks in persistent systems, and for long outburst durations and recurrence times in transients. The geometry of irradiation, whether direct or indirect, and the resultant temperature structure, remain uncertain, however. We present the first results from a stucly of irradiation in EXO 0748-676, based on fast UV spectroscopy with $H S T$, X-ray spectral and timing information from $R X T E$, fast optical photometry with Gemini-S, and optical spectroscopy with the CTIO $4 \mathrm{mll}$.

We use HST/STIS UV spectroscopy together with optical spectra obtained to construct an optical/UV spectral energy distribution (SED). We measure reddening by fitting the $2175 \AA$ interstellar absorption feature, deriving a very low value, $E(B-V)=0.06 \pm 0.03$. The dereddened SED is shown in Fig. 1. It is strongly peaked and very different to the canonical $\nu^{1 / 3}$ disk spectrum. Instead it resembles models of irradiated disks, and

\footnotetext{
${ }^{1}$ Astronomy Department and McDonald Observatory, The University of Texas at Austin, USA

${ }^{2}$ Hubble Fellow; rih@astro.as.utexas.edu

${ }^{3}$ Department of Physics and Astronomy, The Open University, UK

${ }^{4}$ School of Physics and Astronomy, The University of St Andrews, UK

${ }^{5}$ European Southern Observatory

${ }^{6}$ School of Physics and Astronomy. The University of Southampton, UK

${ }^{7}$ Department of Physics and Astronomy, The University of Leicester, UK
}

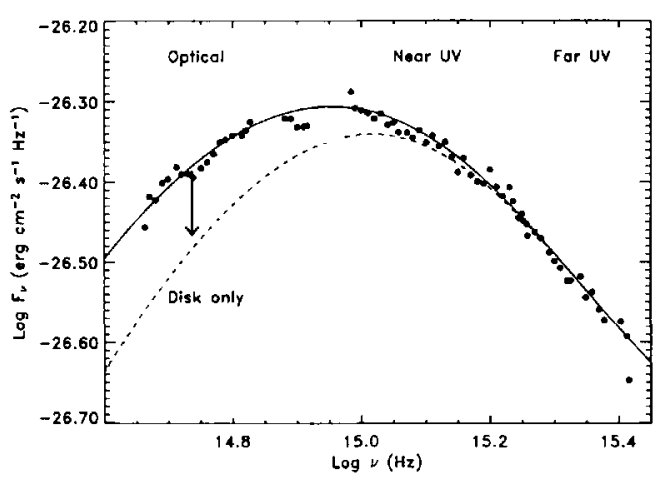

Fig. 1. Optical/UV SED of EXO 0748-676. The dashed line shows an irradiated disk model with edge temperatures of $T_{\mathrm{irr}}=11,500 \mathrm{~K}$ and $T_{\mathrm{vis}}=4200 \mathrm{~K}$. The solid line shows the effect of adding an $8000 \mathrm{~K}$ black body component representing the heated companion star. This is intended to be a representative model rather than a full fit, and other decompositions are possible.

in particular the HST spectrum of XTE J1859+226 (Hynes et al. 2002). The peak is at lower frequencies, however, suggesting that the edge of the disk is cooler. An additional factor that must be accounted for, however, is heating of the companion star. This would be expected to introduce a quasiblackbody spectrum possibly sharpening the peak of the SED. In the case of EXO 0748-676, we have optical and UV orbital lightcurves at the time of our observations. We do see a weak heating effect in the optical lightcurve but none in the UV, suggesting that at maximum light, around phase 0.5 , the 
companion contributes about $20 \%$ of the optical flux and negligible UV flux. Fig. 1 shows a representative irradiated disk plus black body model consistent with both the optical/UV SED and the requirement that the companion contribute $\sim 20 \%$ of the optical light. The disk is modeled following Hynes et al. (2002) with an irradiative heating edge temperature of $T_{\mathrm{irr}}=11,500 \mathrm{~K}$ and a viscous heating temperature of $T_{\text {vis }}=4,200 \mathrm{~K}$. Note that the steepness of the far-UV dropoff requires a low viscous temperature; values as high as $6,000 \mathrm{~K}$ are inconsistent with the data. This suggests that the disk cannot be maintained in a hot state by viscous heating alone, and that it is being stabilized by irradiation.

Optical and UV bursts more directly isolate the reprocessed component, and light travel times provide further spatial information on the reprocessing geometry. We model optical/UV bursts based on the observed X-ray burst profile. We formulate the problem in a similar way to Hynes et al. (1998), but with some extensions. Rather than relating X-ray and optical/UV lightcurves directly, we estimate an X-ray fiux lightcurve, and then use this as the bolometric irradiating luminosity, convolved with a Gaussian transfer function, to determine the bolometric reprocessed flux. From this, the effective temperature evolution, and hence the optical and UV lightcurves, are derived. This procedure is only constrained in this case because the optical and UV bandpasses are sufficiently separated to constrain the burst temperature spectrally. We tried to fit this model to the burst data assuming a single black body reprocessor, but could not simultaneously fit both optical and UV lightcurves without arbitrarily renormalizing one of the lightcurves by more than a factor of two. We therefore instead considered two component models. Two black body components allow an adequate fit, but we instead use an irradiated disk plus a black body, for consistency with the SED modeling. We fix pre-burst temperatures at the values found from the SED, and allow emitting area and peak temperature to vary together with the Gaussian transfer function of each component. We fird the companion star heated from $8,000 \mathrm{~K}$ to $16,000 \mathrm{~K}$ and the disk edge irradiation temperature rising from $11500 \mathrm{~K}$ to $26,000 \mathrm{~K}$. The fitted lightcurves are shown in Fig. 2. Like the models for the SED, these are representative models consistent with the data, but may not be unique solutions.

We can compare the temperatures estimated above to predictions of a toy model of EXO 0748.676. The out-of-burst luminosity corresponds to a mass transfer rate of about $4 \times 10^{16} \mathrm{~g} \mathrm{~s}^{-1}$ and hence

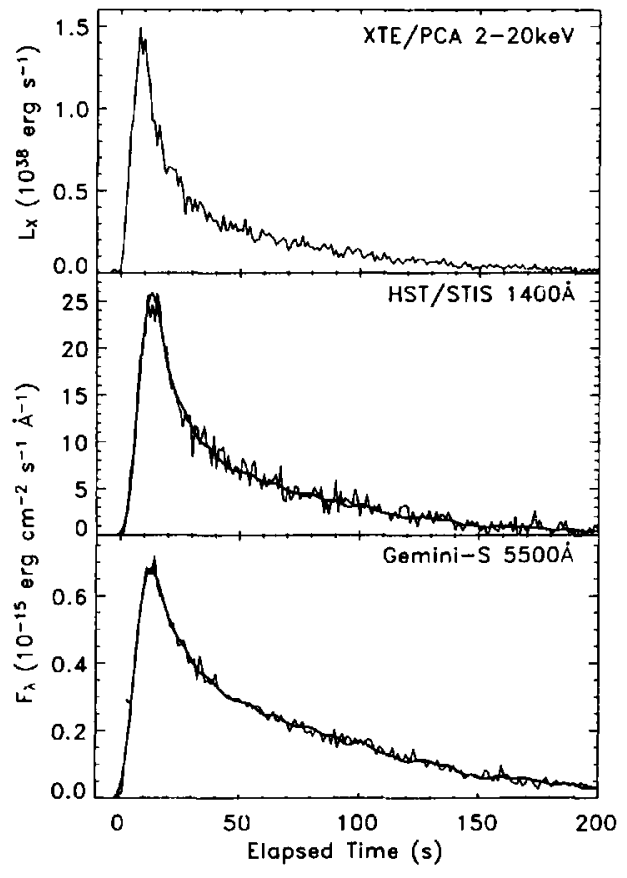

Fig. 2. Simultaneous burst profiles. Fits to the UV/optical data are described in the text.

an edge viscous temperature of $3,300 \mathrm{~K}$. Assuming the irradiation prescription of Dubus et al. (2001) we then estimate out-of-burst and burst-peak disk irradiation temperatures of $10,000 \mathrm{~K}$ and $22,000 \mathrm{~K}$ respectively. For the companion if we assume an $\mathrm{X}$ ray albedo of $\sim 0.5$ and $45^{\circ}$ angle of incidence we expect out-of-burst and burst-peak temperatures of $22,000 \mathrm{~K}$ and $47,000 \mathrm{~K}$ respectively. The parameters we earlier obtained for the disk are thus very sensible, and the toy model supports the conclusion that viscous heating alone is insufficient to keep the disk in a hot state. We do not see evidence for the extremely strong heating of the companion which is expected, however. It may be that the companion is small enough to be largely shielded by the disk rim, so that only the polar caps are exposed to irradiation at a steep angle of incidence.

This work includes observations made with $H S T$, RXTE, Gemini, and CTIO. RIH is supported by NASA through a Hubble Fellowship.

\section{REFERENCES}

Dubus, G., Hameury, J.-M., \& Lasota, J.-P. 2001, A\&A. 373,251

Hynes, R. I., Haswell, C. A., Chaty, S., Shrader, C. R.. \& Cui, W. 2002, MNRAS, 331, 169

Hynes, R. I., O'Brien, K., Horne, K., Chen, W. \& Haswell, C. A. 1998, MNRAS, 299, L37 